\title{
The importance of airway and lung microbiome in the critically ill
}

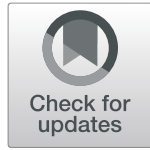

\author{
Ignacio Martin-Loeches ${ }^{1,2,3^{*}}$ (D, Robert Dickson ${ }^{4,5,6}$, Antoni Torres ${ }^{7}$, Håkan Hanberger ${ }^{8,9}$, Jeffrey Lipman ${ }^{10,11}$, \\ Massimo Antonelli ${ }^{12,13}$, Gennaro de Pascale ${ }^{12,13}$, Fernando Bozza ${ }^{14}$, Jean Louis Vincent ${ }^{15}$, Srinivas Murthy ${ }^{16}$, \\ Michael Bauer ${ }^{17}$, John Marshall ${ }^{18}$, Catia Cilloniz ${ }^{7}$ and Lieuwe D. Bos ${ }^{19}$
}

\begin{abstract}
During critical illness, there are a multitude of forces such as antibiotic use, mechanical ventilation, diet changes and inflammatory responses that could bring the microbiome out of balance. This so-called dysbiosis of the microbiome seems to be involved in immunological responses and may influence outcomes even in individuals who are not as vulnerable as a critically ill ICU population. It is therefore probable that dysbiosis of the microbiome is a consequence of critical illness and may, subsequently, shape an inadequate response to these circumstances. Bronchoscopic studies have revealed that the carina represents the densest site of bacterial DNA along healthy airways, with a tapering density with further bifurcations. This likely reflects the influence of micro-aspiration as the primary route of microbial immigration in healthy adults. Though bacterial DNA density grows extremely sparse at smaller airways, bacterial signal is still consistently detectable in bronchoalveolar lavage fluid, likely reflecting the fact that lavage via a wedged bronchoscope samples an enormous surface area of small airways and alveoli. The dogma of lung sterility also violated numerous observations that long predated culture-independent microbiology. The body's resident microbial consortia (gut and/or respiratory microbiota) affect normal host inflammatory and immune response mechanisms. Disruptions in these host-pathogen interactions have been associated with infection and altered innate immunity.

In this narrative review, we will focus on the rationale and current evidence for a pathogenic role of the lung microbiome in the exacerbation of complications of critical illness, such as acute respiratory distress syndrome and ventilator-associated pneumonia.
\end{abstract}

Keywords: Pneumonia, Microbiome , Infection, Ventilator-associated pneumonia, Ventilator-associated tracheobronchitis

\section{Introduction}

The normal microbiota is the ecological communities of commensal, symbiotic and pathogenic microorganisms whilst the microbiome comprises all of the genetic material within a microbiota (the entire collection of microorganisms

\footnotetext{
* Correspondence: drmartinloeches@gmail.com

'Department of Intensive Care Medicine, Multidisciplinary Intensive Care Research Organization (MICRO), St James Hospital, Dublin 8., Ireland ${ }^{2}$ Department of Respiratory Medicine, Hospital Clinic, IDIBAPS, CIBERes, Barcelona, Spain

Full list of author information is available at the end of the article
}

in a specific niche, such as the human gut). This can also be referred to as the metagenome of the microbiota [1, 2]. Approximately 100 billion microorganisms are found in the body due to recent discoveries in molecular analysis such as next-generation sequencing (NGS) and whole metagenome shotgun sequencing (WMGS); there is an increasing body of evidence pointing towards the dysbiosis that is often defined as an 'imbalance' in the microbial community that is associated with disease [3-5].

A microbiome is shaped by multiple factors including the resident flora of the animate or inanimate vicinity 
and the external forces that modulate this flora [6]. It becomes a changeable reflection of diversity, and so its study can provide valuable insights into the factors that drive that diversity [7]. Just as the study of global climate or the roots of language requires input from around the world, so the interpretation of the microbiome of an individual or a group of patients needs comprehensive comparative data to generate insight $[8,9]$. The variability of the host microbiome-either in an individual patient over time in response to the pressures of illness [10] or in a geographically localized population in response to environmental-can yield important insight into factors that can be manipulated to improve clinical outcomes. Such factors include risk of infection, emergence of resistance, spread from the environment, host susceptibility and even the resilience of the health care system [11].

In this narrative review, we will focus on the rationale and current evidence for a pathogenic role of the lung microbiome in the exacerbation of complications of critical illness, such as acute respiratory distress syndrome (ARDS) and ventilator-associated pneumonia (VAP).

\section{Is the lung sterile or not?}

Though for years textbooks taught that 'the normal lung is free from bacteria', this dogma was generally repeated without citation or argument [12]. In retrospect, this claim of lung sterility was remarkable: virtually no environment on earth exists that is so extreme in temperature, $\mathrm{pH}$, salinity or nutrient scarcity that microbial communities cannot be detected [13]. Yet for more than a century, it was taken as fact that the warm, wet mucosa of the lower respiratory tract-mere inches below the microbial reservoir of the pharynx-is an exception to this rule [14-18].

Each individual has a unique microbiota profile that plays many specific functions in host nutrient metabolism, maintenance of structural integrity and protection against pathogens. There is not a unique optimal microbiota composition as it can be different for each individual $[19,20]$. Thus, the 'revolution' in culture-independent microbiology has merely confirmed with certainty what has long been inferred indirectly: human lungs are constantly exposed to environmental bacteria. To date, more than 30 studies have used sensitive, culture-independent techniques to study lung bacteria in healthy volunteers, and none has failed to detect a distinct bacterial signal [21]. The viability of bacteria in healthy lungs has been confirmed via advanced cultivation [22] and indirectly validated via correlation with healthy alveolar immune tone in humans and mice [23, 24].

Some of the confusion regarding the existence of lung microbiota reflects flawed parallels with the lower gut microbiome, which represents a wholly different ecosystem with radically different ecologic forces. Whereas the gut lumen is densely populated by dense communities' bacteria, lung microbiota is scarce and associated with mucosal surfaces. Whereas gut communities are relatively stable dayto-day, reflecting stable selective pressure on resident bacteria, lung communities are in constant turnover, with their identities and burdened determined by the relative balance of immigration (via microaspiration and mucosal dispersion) and elimination (via cough and mucociliary clearance). Whereas the gut microbiome is nutrient-rich and characterized by intense metabolic competition amongst dense communities, the lung microenvironment is nutrient-poor, and the primary competition is between immigrating pharyngeal microbes and locally calibrated alveolar and airway host defences attempting to minimize their outgrowth [24, 25]. These ecologic differences between the lower gut and the lungs erode somewhat in conditions of acute and chronic disease: the influx of mucus and proteinrich oedema provide nutrient sources for bacteria, and once-transient bacteria become resident, shaped by selective pressure.

Further confusion arose via misinterpretation of clinical culture protocols, which have been optimized for detection of respiratory pathogens, not the 'background' microbiota of uninfected patients. Sequencing-based studies have revealed that the normal microbiota of healthy lungs closely resembles that of the oropharynx [26-28] and, whilst commonly cultured, are routinely dismissed by clinical microbiology laboratories as 'normal oral flora'.

Bronchoscopic studies have revealed that the carina represents the densest site of bacterial DNA along healthy airways, with a tapering density with further bifurcations [28]. This likely reflects the influence of micro-aspiration as the primary route of microbial immigration in healthy adults. Though bacterial DNA density grows extremely sparse at smaller airways, bacterial signal is still consistently detectable in bronchoalveolar lavage fluid, likely reflecting the fact that lavage via a wedged bronchoscope samples an enormous surface area of small airways and alveoli. Bacterial communities within the lungs of healthy volunteers are relatively homogenous; the bacteria of a given individual's right middle lobe far more closely those of the same individual's left upper lobe than do other individuals' right middle lobe (i.e. intraindividual similarity is greater than interindividual similarity) [27].

\section{How to study the lung microbiome?}

High densities of bacteria are always present on the skin, in the mouth, and in the upper respiratory tract. For this reason, it is important to avoid contamination with commensal bacteria from other sites when taking samples for investigation of the lower respiratory tract microbiome 
$[29,30]$. Since samples from the lower respiratory tract may have a low biomass, it increases the risk for contamination that can occur at any time from sampling to sequencing [31, 32].

The first molecular techniques used for studying the bacterial microbiome in humans were based on $16 \mathrm{~S}$ rRNA gene sequencing many years ago which is an appropriate method to assess diversity on taxonomic levels above species level. A limitation of 16S rRNA gene sequencing is that whilst bacteria can normally be identified on genus and family level, species identification usually requires simultaneous evaluation of several genes [33-35]. Newer technology of whole genome sequencing and metagenomics has shown better definition of the gut microbiome and what has currently been shown of the lung microbiome will also be significantly updated by these newer sequencing technologies [36-38].

An important matter is that when studying the lung microbiome, the pathogens and host response needs to be simultaneously studied by molecular methods, for instance, microbial metagenomics and transcriptomics. Langelier et al. [39] performed in almost 100 patients with acute respiratory failure (ARF) metagenomic nextgeneration sequencing (mNGS) on endotracheal aspirates (ETA) and simultaneously assessed pathogens, the airway microbiome and the host transcriptome. This study found that a single streamlined protocol offering an integrated genomic portrait of pathogen, microbiome and host transcriptome represents a new tool for diagnosis in lower respiratory tract infections (LRTI).

The progress in molecular microbiology has developed very fast in the last years and several rapid technologies will provide biological signals taking into account the interaction of the host (e.g. via digital enzyme-linked immunosorbent assay (ELISA) [40]) and the microbes (e.g. via nanorod-PCR [41]). Another technology is microgas chromatography for the analysis of bacterial function and virulence and metabolic indices of the host response on exhaled breath $[42,43]$.

The field of lung microbiome is no longer limited by the speed of sequencing, processing, or measurement, but rather our ability to make sense of the highdimensional data we generate.

\section{Lung microbiome in ARDS}

ARDS is a complication of critical illness characterized by protein-rich pulmonary oedema, hypoxaemia and alveolar inflammation. Alveolar inflammation, damage and subsequent oedema may be initiated by a change in pulmonary microbiome, or a change in lung microbiome may be initiated by an alveolar nutrient available after the onset of oedema [2]. Even though ARDS is traditionally not considered to be related to microbial changes in the lung, these physiological considerations resulted in the hypothesis that pathogenic bacteria may be present in the lung of patients with ARDS.

Kyo et al. [44] analysed the lung microbiome from the bronchoalveolar lavage fluid (BALF) of patients with ARDS found that lung bacterial burden (16S rRNA gene copy numbers tended to be increased) tended to be increased, and the alpha diversity (copy numbers and relative abundance of betaproteobacteria) was significantly decreased in ARDS patients.

In an experimental mouse model of lung injury following abdominal sepsis induced by cecal ligation and puncture, the lung microbiome was enriched with gut bacteria [3]. How did these bacteria get there? It is hypothesized that bacteria can translocate from the gut into the lymphatic system and portal circulation during critical illness [4]. If so, these changes should also be observed in patients on the ICU. Indeed, enrichment of gut bacteria was also observed in BALF from ARDS patients [3]. Gut bacteria and more specifically Enterobacterieae enrichment in patients with ARDS were confirmed in a second observational cohort study [5]. Both studies were performed in a selective cohort of patients with potential biases of prolonged antibiotic exposure before measurement. In a more recent study conducted in Europe, patients who were treated with selective decontamination of the digestive tract (SDD) during admission at the ICU, but were not treated with antibiotics prior to ICU admission, validated the specific enrichment of Enterobacterieae in the lungs of ARDS patients [45].

Taken together, the current body of evidence suggests that amplification of Enterobacterieae in the lung is strongly associated with ARDS. This association is not sufficiently explained by potential confounders such as geographical location of sampling, exposure to antibiotic therapy, amplification protocols or exact definitions of ARDS. The evidence for consistent dysbiosis in lung microbiome is actually stronger for ARDS than for most other respiratory diseases, where other microbes are enriched in different studies. However, no causal link between dysbiosis of the lung microbiome and development of lung injury has been established. This link needs to be further explored before we can conclude that lung microbiome dysbiosis is a potential target for treatment (Fig. 1).

\section{Lung microbiome during nosocomial LRTI}

In ecological terms, pneumonia can be described as the collapse of local microbiome diversity and the emergence of a dominant pathogen [46]. Several studies have therefore hypothesized that the lung changes considerably during nosocomial lower respiratory tract infections. Some critically ill patients can develop pneumonia due to their clinical condition such as patients with ischaemic stroke and/or with loss of neurological control 


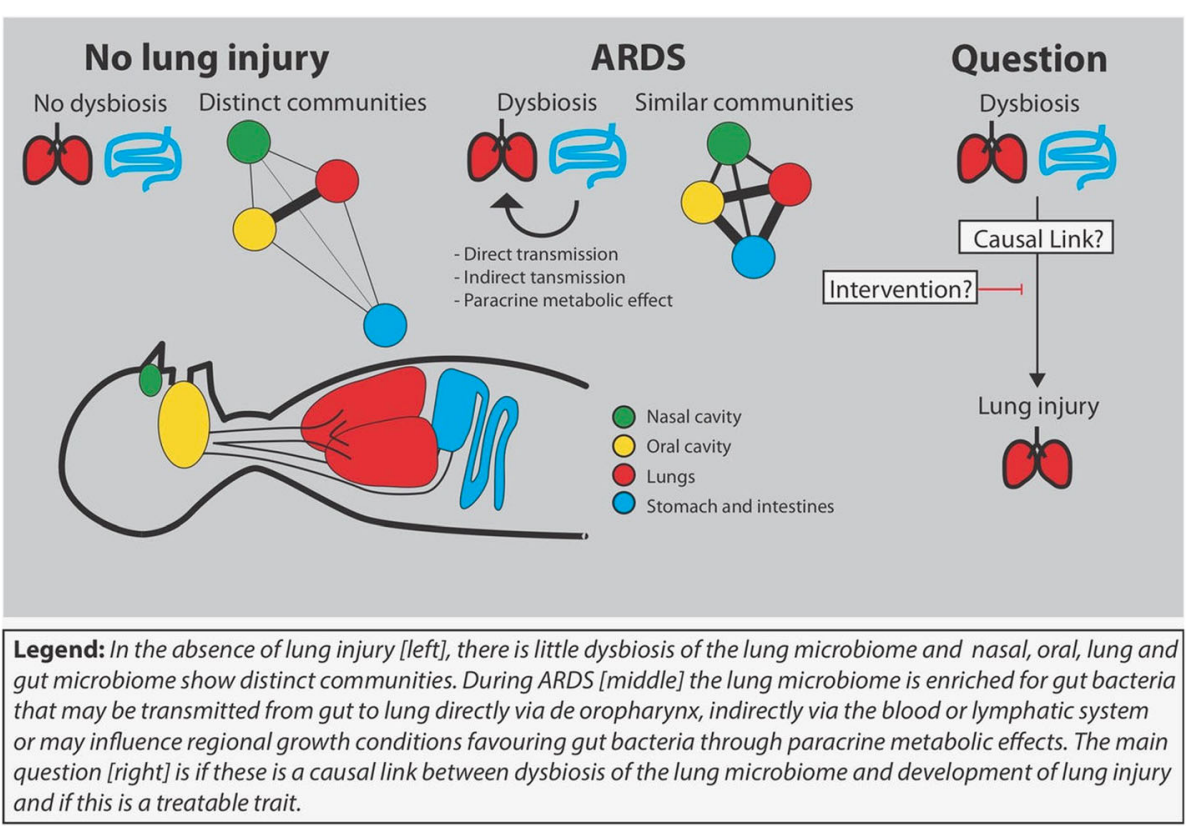

Fig. 1 Algorithm of dysbiosis pathways in patients with pneumonia, ARDS and influence of mechanical ventilation. ARDS, acute respiratory distress syndrome

of the respiratory system. These clinical conditions can be associated to reduced airway clearance and increased bacterial translocation and therefore can develop more often respiratory infections [47]. So, the more appropriate question is 'do patients that develop pneumonia have more dysbiosis of the lung microbiome than mechanically ventilated ICU patients who do not develop pneumonia'? Two studies addressed this problem. The first included consecutive patients at risk for pneumonia with a duration of mechanical ventilation of more than 7 days [48]. Endotracheal aspirates were performed every third day and the microbial composition was evaluated with $16 \mathrm{~S}$ sequencing. There was a small, but significant increase in the change in beta-diversity (change in diversity of species from one environment to another) in patients who went on to develop pneumonia as compared to patients who did not develop any signs of infection and were not colonized by any bacteria according to traditional bacterial cultures. The composition of the microbiome in these patients also showed a slight enrichment of Pseudomonadales. A second study conducted had a similar design and showed no difference in the change of microbiome during mechanical ventilation between patients who did and did not develop pneumonia [49]. As discussed in the accompanying editorial, the results from these studies have elegantly shown that it is time to let go of any simplistic view of VAP pathogenesis [10]. One conclusion might be that LRTI cannot simply be defined as a collapse of bacterial ecology as this is present also in part of the patients without pneumonia who do not show any signs of pneumonia. One could also argue that the studies did not sample the alveolar space and additional studies with BALF are needed to confirm or discard these findings. Furthermore, evaluation of microbial composition may be more useful in establishing the presence of a pathogen in patients who already have a clinical suspicion of pneumonia. Indeed, with pre-test probability, metagenomics may provide valuable information on the pathogen causing pneumonia [11]. Future studies have to consider these possibilities before we disregard the lung microbiome in nosocomial pneumonia.

\section{Gut-lung axis and the possible interventions}

In the critically ill, changes in the microbiome in all habitats, including the lungs, are particularly striking. Due to the devastating consequences of untreated severe infections, broad eradication is accepted as lesser evil and collateral damage on beneficial or commensal microbes is generally accepted. However, the potential long-term consequences of unwarranted side effects on the microbiome warrant a reassessment of the microbiome as a diagnostic or even therapeutic target. For example, dysbiosis of the gut microbiome itself has been described as a predictive factor for late-onset neonatal sepsis [50] suggesting that the microbiome can serve at least as a biomarker to predict ensuing nosocomial infection. Moreover, albeit solid data are still missing to support interventions to restore a healthy microbiome, the strategy holds promise to impact on incidence and outcome of nosocomial infection and ensuing organ injury, 
including ARDS [51, 52]. In the light of a better understanding of off-target effects of broad-spectrum antibiotics on the microbiome, the liberal administration of antibiotics must be discussed against more sophisticated interventions to treat the bacterial infection (non-antibiotic therapies such as bacteriophages) or manipulation of the microbiome to make the residing communities more resilient (for example probiotics). In particular, the need to combine multiple anti-infective compounds in the light of diagnostic uncertainty might outweigh the benefit of early source control and explain controversial results for aggressive antibiotic strategies. For instance, in a before-andafter study Hranjec et al. reported that the subgroup with least benefit from 'calculated' broad-spectrum antibiotics were patients presenting with septic shock, i.e. those in which the current paradigm would expect the highest need to initiate early anti-infective therapy [53]. Thus, a holistic approach taking the microbiome into consideration carries the potential to initiate a paradigm shift in the treatment of infections in the ICU.

As discussed in the previous paragraphs, the lung dysbiosis seems to be common in the ICU and enrichment of gut bacteria might be an important contributor to the development of lung injury and infection (Fig. 2). The relationship between gut and lung microbiome is described as the gut-lung axis [54]. Because the gut microbiome can be targeted directly or indirectly with therapeutic interventions, this is an area of active study. Investigations have thus far fallen into two specific pathways-first, using probiotics to help restore a premorbid microbiome, or second, to use antibiotics through an SDD approach to target specific families of organisms so as to alter the microbiome in possibly beneficial ways. Further novel pharmacologic options that have direct gut microbiome modifying effects are also under development, including faecal transplantation as a possible novel treatment for microbiota dysregulation (considering the immune system during faecal microbiota transplantation for Clostridioides difficile infection [55] and for the decolonization of antibioticresistant bacteria in the gut [56]). One of the major challenges of studying the effect of these interventions is the huge variability in the gut microbiome of critically ill patients, even during the first days of ICU admission [57]. Furthermore, any beneficial effect of these interventions on the microbiome has yet to be assessed formally in a prospective, large-scale, randomized manner. Attempting to attribute a causal impact of microbiome modifications upon clinical outcomes has been difficult to tease out as to whether changes in the microbiome are merely surrogates of some other mechanistic pathway that leads to improved clinical outcomes [58].

\section{Probiotics}

Fundamentally, probiotics in critical illness aim to provide bacteria that may have been eradicated during the pre- and early phases of critical illness [59]. This eradication may be through administering antibiotics early in critical illness, which have been shown to greatly modify the gut microbiome [60]. Alternatively, the mere onset of critical illness-be it sepsis, ARDS or any number of conditions, is associated with alterations of the gut

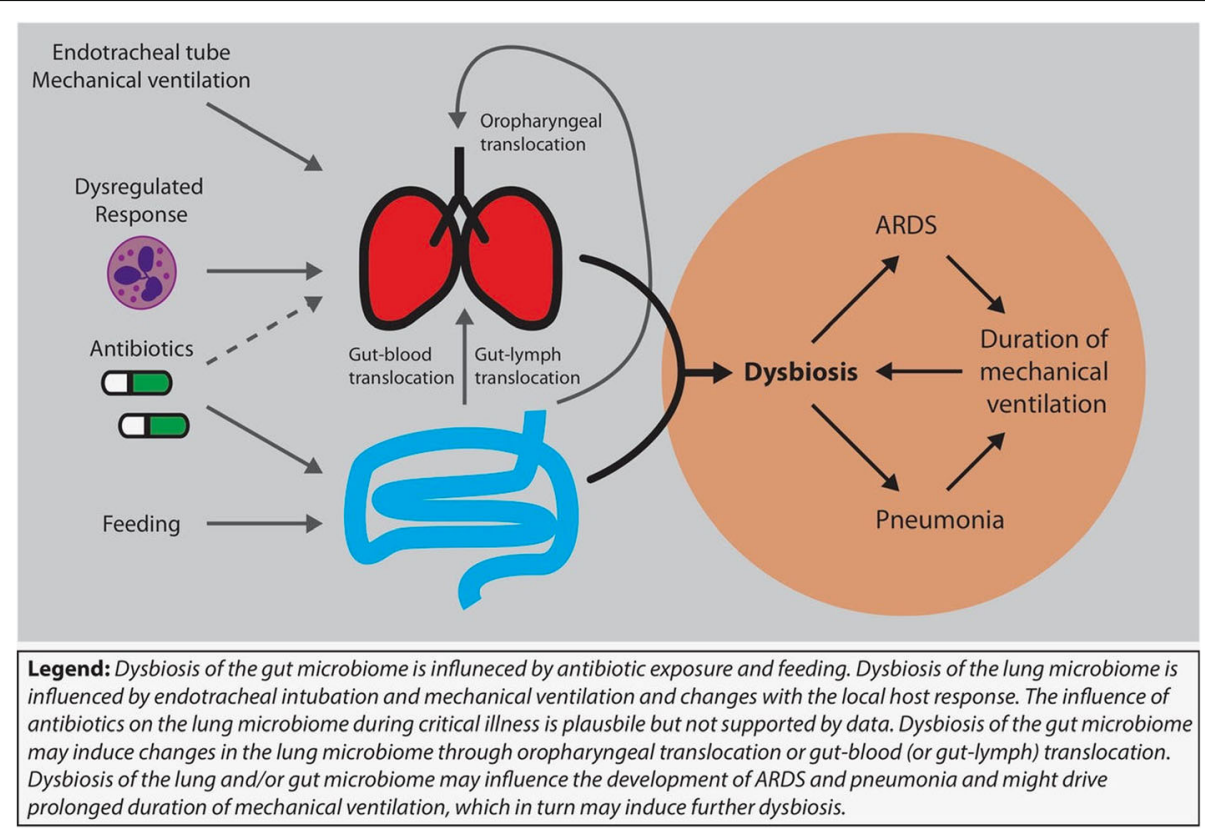

Fig. 2 Island model for the development of lung injury based on sites of dysbiosis 
microbiome, which may be independent of antibiotic administration [61]. Regardless, the stated goal of probiotic administration is to restore a pre-morbid microbiomeprimarily to the gut, but partially to other microbiome communities through generalized cross-talk [62]. Through yet-unknown mechanisms, administering Lactobacillus or Bifidobacterium species through a probiotic may increase the diversity of microbial species in the gut, although more studies with rigorous outcome determinations are required [63]. In the critically ill, randomized studies and meta-analyses of randomized trials demonstrate a possible benefit of probiotic administration on the outcome of ventilator-associated pneumonia, without a difference in mortality $[64,65]$, with a major challenge being a lack of standardization in dosing and composition of probiotic products [66]. Larger scale studies are nearing completion and further data on the impact of microbiome modifications are forthcoming in the years ahead [59].

\section{Selective digestive decontamination}

Selective digestive decontamination, a regimen of prophylactic antibiotic administration, has been shown in small series to result in important alterations in gut microbiota, when compared with controls [67]. These changes are typically related to increasing selection for resistant organisms and decreased microbiome diversity, per a number of different metrics. Given a possible benefit on patient mortality in some randomized trials $[68,69]$, exploring the specific impact of this strategy on the microbiome, and related clinical outcomes, is a vital area for further study. Additionally, given burgeoning evidence of crosstalk between the lung and gut microbial communities, the impact of either of these strategies on the non-gut microbiome communities in the critically ill patient remains under-investigated.

Given the apparent conflicting goals of SDD and probiotic administration in the critically ill as it relates to the microbiome, the role of co-administration may be difficult to conceive. However, most currently used SDD regimens are unlikely to affect the administered probiotic agent, and this may be a strategy for further investigation in targeted patients [70]. Both SDD and probiotics appear to mediate their effect on patientrelated outcomes through reducing the incidence of ventilator-associated pneumonia, speaking to a crucially under-investigated relationship between the two microbiome communities and host immunology, a tantalizing area for future research.

\section{Other treatments}

Novel pharmacologic agents have also been suggested as modifiers for the gut microbiome but have yet to be formally tested in the critically ill. Butyrate, a large bowel microbial fermentation product, is being investigated in pre-clinical trials as a specific modifier of gut-derived regulatory $\mathrm{T}$ cells [71]. Administering a sialic acid analogue is being investigated as to whether it may reduce the burden of antibiotic-associated pathogens such as $C$. difficile by altering metabolic pathways [72]. Older drugs such as metformin may have a role, with their demonstrated effects on altering the gut microbiome in patients with diabetes [73].

The lung microbiome is clearly more difficult to target than the gut microbiome due to the lack of routine administration of bacteria and bacterial products into the airways. The low biomass environment may also cause the lung microbiome to be more prone to infection induced by the introduction of, for example, probiotics. Therefore, direct intervention in the lung microbiome may be sought via the alteration of regional growth conditions via the availability of nutrients or through immunomodulation. An example is the administration of macrolides in chronic obstructive pulmonary disease (COPD): there is a selection for anti-inflammatory microbial metabolites and an alteration of the lung microbiome [74].

All of these possible interventions speak to the importance of achieving a better understanding of the gut-lung axis in critical illness. As this understanding evolves, the possibility of personalizing interventions for individual microbiome communities, or widespread initiation of interventions such as SDD or probiotics, would be possible.

\section{The need for a network to support activities}

Whilst patient-to-patient or staff-to-patient transmission of infection occurs within the intensive care unit, most nosocomial infections in critically ill patients arise through the invasion of normal host defences by bacteria and fungi that have become a part of an altered microbiome-either by changes in numbers or by the incorporation of species from the environment [75]. The hospital environment itself acquires a microbiome that reflects the patients that have been in it, and environmental reservoirs such as sinks, plumbing, work surfaces, and equipment can become reservoirs of resistant organisms that can infect the critically ill [76].

The inherent variability of the microbiome, therefore, provides an opportunity to study not only the individual patient, but also the forces in the environment that shape patient's outcome, and to identify specific opportunities where the persistence and transmission of pathogens can be prevented or minimized. Because of the high prevalence of nosocomial infection, the environmental concentration of causative pathogens and the multiple risk factors for exposure, the ICU provides a unique opportunity for intensive study of the microbiome and its role in the establishment and transmission 
of resistant organisms. With the emergence of new models of global acute care research collaboration through the International Forum for Acute Care Trialists (InFACT; www.InFACTglobal.org), and the launch of an InFACT initiative to leverage ICU data to understand variability in patterns of resistance through the Antimicrobial Resistance in Intensive Care (AMRIC) initiative.

\section{Conclusion}

In previous years, we believed that the normal lung was free from bacteria. Certainly, some features in the respiratory tract such as temperature, $\mathrm{pH}$ and nutrients were not beneficial for microbial growth. During critical illness, antibiotic use, mechanical ventilation, diet changes and inflammatory responses can bring the microbiome to dysbiosis.

With the use of molecular techniques, we have had the opportunity to study the lung microbiome and not only in the microbial aspect but also in the responses from the host. One of the most important aspects to better determine the physiopathology of host-pathogen interaction in pulmonary complications such as ARDS and VA-LRTI is the gut-lung axis. Further study of patients with disease in the respiratory tract will help us to better determine microbial diversity and constitution when comparing healthy and diseased subjects.

Dysbiosis and analysis of extra-pulmonary microbiome have helped to understand the complex interaction of bacterial clearance in the lung tissue and the off-target effects of broad-spectrum antibiotics on the microbiome.

Through therapies targeting host-pathogen interaction and the development of advance molecular testing, we will be able to have a deeper understanding in the analysis of the lung microbiome.

Acknowledgements

Not applicable.

\section{Authors' contributions}

IML has contributed to the conception of the manuscript. RD, AT, HH, JL, $M A, G d P, F B, J L V, S M, M B, J M, C C$ and LB drafted different sections of the manuscript. The authors read and approved the final manuscript.

\section{Funding}

Ignacio Martin-Loeches is supported by the Health Research Board (HRB)/Joint Programming Initiative on Antimicrobial Resistance (JPIAMR) (2018-06335). Michael Bauer is supported by Deutsche Forschungsgemeinschaft (DFG, German Research \& Foundation) under Germany's Excellence Strategy-EXC 2051_-Project-ID 390713860).

Availability of data and materials

Attached to the manuscript.

Ethics approval and consent to participate Not applicable.

Consent for publication

Obtained by all the authors.

\section{Competing interests}

No COls to declare.

\section{Author details}

${ }^{1}$ Department of Intensive Care Medicine, Multidisciplinary Intensive Care Research Organization (MICRO), St James Hospital, Dublin 8., Ireland. ${ }^{2}$ Department of Respiratory Medicine, Hospital Clinic, IDIBAPS, CIBERes, Barcelona, Spain. ${ }^{3}$ Trinity College, Dublin, Ireland. ${ }^{4}$ Division of Pulmonary and Critical Care Medicine, Department of Internal Medicine, University of Michigan, Ann Arbor, USA. ${ }^{5}$ Department of Microbiology and Immunology, University of Michigan, Ann Arbor, USA. ${ }^{6}$ Michigan Center for Integrative Research in Critical Care, Ann Arbor, MI, USA. ${ }^{7}$ Deparment of Pneumology, Institut Clinic del Tórax, Hospital Clinic of Barcelona - Institut d'Investigacions Biomèdiques August Pi i Sunyer (IDIBAPS), University of Barcelona (UB) - SGR 911- Ciber de Enfermedades Respiratorias (Ciberes), Barcelona, Spain.

${ }^{8}$ Department of Infectious Diseases, Linköping University, Linköping, Sweden. ${ }^{9}$ Department of Clinical and Experimental Medicine, Linköping University, Linköping, Sweden. ${ }^{10}$ The University of Queensland, Brisbane, Australia. ${ }^{11}$ Scientific Consultant, Nimes University Hospital, University of Montpellier, Nimes, France. ${ }^{12}$ Department of Anesthesiology, Intensive Care and Emergency Medicine, Fondazione Policlinico Universitario A. Gemelli IRCCS, Rome, Italy. ${ }^{13}$ Università Cattolica del Sacro Cuore, Rome, Italy. ${ }^{14}$ National Institute of Infectious Diseases Evandro Chagas, Oswaldo Cruz Foundation, Fiocruz, Rio de Janeiro, Brazil. ${ }^{15}$ Department of Intensive Care, Erasme University Hospital, Université Libre de Bruxelles, Brussels, Belgium.

${ }^{16}$ University of British Columbia, Vancouver, BC V6H 3V4, Canada.

${ }^{17}$ Department of Anesthesiology and Intensive Care Medicine, Jena University Hospital, Am Klinikum 1, 07747 Jena, Germany. ${ }^{18}$ The Keenan Research Centre for Biomedical Science, The Li Ka Shing Knowledge Institute, St Michael's Hospital, University of Toronto, Toronto, Ontario, Canada.

${ }^{19}$ Department of Respiratory Medicine, Infection and Immunity, Amsterdam University Medical Center, AMC, Amsterdam, The Netherlands.

Received: 5 May 2020 Accepted: 3 August 2020

Published online: 31 August 2020

\section{References}

1. Ikeda-Ohtsubo W, Brugman S, Warden CH, Rebel JMJ, Folkerts G, Pieterse CMJ. How can we define "optimal microbiota?": a comparative review of structure and functions of microbiota of animals, fish, and plants in agriculture. Front Nutr. 2018;5 Available from: https://www.frontiersin.org/ article/10.3389/fnut.2018.00090/full. Accessed 15 June 2020.

2. Ruff WE, Greiling TM, Kriegel MA. Host-microbiota interactions in immunemediated diseases. Nat Rev Microbiol. 2020:1-18 Available from: http:// www.nature.com/articles/s41579-020-0367-2. [cited 2020 Jun 4].

3. Weinstock GM. Genomic approaches to studying the human microbiota. Nature. 2012;489:250-6 Available from: http://www.nature.com/articles/ nature11553.

4. Petersen $C$, Round $J$. Defining dysbiosis and its influence on host immunity and disease. Cell Microbiol. 2014;16(7):1024-33. https://doi.org/10.1111/cmi. 12308. Epub 2014 Jun 2.

5. Marchesi JR, Ravel J. The vocabulary of microbiome research: a proposal. Microbiome. 2015;3:31 Available from: https://microbiomejournal. biomedcentral.com/articles/10.1186/s40168-015-0094-5. [cited 2020 Jun 4].

6. Grice EA, Segre JA. The human microbiome: our second genome. Annu Rev Genomics Hum Genet. 2012;13:151-70 Available from: http://www. annualreviews.org/doi/10.1146/annurev-genom-090711-163814.

7. Moeller $\mathrm{AH}$, Ochman $\mathrm{H}$. Factors that drive variation among gut microbial communities. Gut Microbes. 2013:4:403-8 Available from: http://www. tandfonline.com/doi/abs/10.4161/gmic.26039.

8. Biteen JS, Blainey PC, Cardon ZG, Chun M, Church GM, Dorrestein PC, et al. Tools for the microbiome: nano and beyond. ACS Nano. 2016;10:6-37 Available from: https://pubs.acs.org/doi/10.1021/acsnano.5b07826.

9. Pasolli E, Asnicar F, Manara S, Zolfo M, Karcher N, Armanini F, et al. Extensive unexplored human microbiome diversity revealed by over 150,000 genomes from metagenomes spanning age, geography, and lifestyle. Cell. 2019;176:649-662.e20 Available from: http://www.ncbi.nlm.nih.gov/ pubmed/30661755.

10. McDonald D, Ackermann G, Khailova L, Baird C, Heyland D, Kozar R, et al. Extreme dysbiosis of the microbiome in critical illness. Green Tringe $\mathrm{S}$, 
editor. mSphere. 2016;1. Available from: http://msphere.asm.org/lookup/ doi/10.1128/mSphere.00199-16. Accessed 15 June 2020.

11. Gaulke CA, Sharpton TJ. The influence of ethnicity and geography on human gut microbiome composition. Nat Med. 2018;24:1495-6 Available from: http://www.nature.com/articles/s41591-018-0210-8.

12. Cotran RS, Kumar V, Collins T, Robbins SL. Robbins pathologic basis of disease, vol. xv. Philadelphia: Saunders; 1999. p. 1425.

13. Horikoshi K, Grant WD. Extremophiles. Microbial Life in Extreme Environments. New York: Wiley-Liss; 1998.

14. Pasteur L. Expériences relatives aux générations dites spontanées. Comptes Rendus Hebdomadaires des Séances de l'Académie des Sciences D: Sciences Naturelles L; 1860. p. 303-7.

15. Dürck H. Studien über die Aetiologie und Histologie der Pneumonie im Kindesalter und der Pneumonie im Allgemeinen. Centr Bakt. 1898;1: 574.

16. Quinn $\mathrm{LH}$, Meyer OO. The relationship of sinusitis and bronchiectasis. Arch Otolaryngol. 1929;10:152-65.

17. Hasleton PS. The internal surface area of the adult human lung. J Anat. 1972;112:391-400 Available from: http://www.ncbi.nlm.nih.gov/ pubmed/4564685.

18. Helander HF, Fändriks L. Surface area of the digestive tract - revisited. Scand J Gastroenterol. 2014;49:681-9 Available from: http://www.ncbi.nlm.nih.gov/ pubmed/24694282.

19. Rinninella E, Raoul P, Cintoni M, Franceschi F, Miggiano GAD, Gasbarrini A, et al. What is the healthy gut microbiota composition? A changing ecosystem across age, environment, diet, and diseases. Microorganisms MDPI AG. Microorganisms. 2019;7(1):14. https://doi.org/10.3390/ microorganisms7010014

20. Qin J, Li R, Raes J, Arumugam M, Burgdorf KS, Manichanh C, et al. A human gut microbial gene catalogue established by metagenomic sequencing. Nature. 2010;464:59-65.

21. Dickson RP, Erb-Downward JR, Martinez FJ, Huffnagle GB. The microbiome and the respiratory tract. Annu Rev Physiol. 2016;78:481-504 Available from: http://www.ncbi.nlm.nih.gov/pubmed/26527186.

22. Venkataraman A, Bassis CM, Beck JM, Young VB, Curtis JL, Huffnagle GB, et al. Application of a neutral community model to assess structuring of the human lung microbiome. MBio. 2015;6 Available from: http://www.ncbi.nlm. nih.gov/pubmed/25604788. Accessed 15 June 2020.

23. Segal LN, Clemente JC, Tsay J-CJ, Koralov SB, Keller BC, Wu BG, et al. Enrichment of the lung microbiome with oral taxa is associated with lung inflammation of a Th17 phenotype. Nat Microbiol. 2016;1:16031 Available from: http://www.ncbi.nlm.nih.gov/pubmed/27572644.

24. Dickson RP, Erb-Downward JR, Falkowski NR, Hunter EM, Ashley SL, Huffnagle GB. The lung microbiota of healthy mice are highly variable, cluster by environment, and reflect variation in baseline lung innate immunity. Am J Respir Crit Care Med. 2018;198:497-508.

25. Dickson RP, Martinez FJ, Huffnagle GB. The role of the microbiome in exacerbations of chronic lung diseases. Lancet. 2014;384:691-702 Available from: http://www.ncbi.nlm.nih.gov/pubmed/25152271.

26. Bassis CM, Erb-Downward JR, Dickson RP, Freeman CM, Schmidt TM, Young VB, et al. Analysis of the upper respiratory tract microbiotas as the source of the lung and gastric microbiotas in healthy individuals. MBio. 2015;6:e00037 Available from: http://www.ncbi.nlm.nih.gov/ pubmed/25736890.

27. Dickson RP, Erb-Downward JR, Freeman CM, McCloskey L, Beck JM, Huffnagle $\mathrm{GB}$, et al. Spatial variation in the healthy human lung microbiome and the adapted island model of lung biogeography. Ann Am Thorac Soc, 12. 2015: 821-30 Available from: http://www.ncbi.nlm.nih.gov/pubmed/25803243. Accessed 15 June 2020

28. Dickson RP, Erb-Downward JR, Freeman CM, McCloskey L, Falkowski NR, Huffnagle GB, et al. Bacterial topography of the healthy human lower respiratory tract. MBio. 2017;8 Available from: http://www.ncbi.nlm.nih.gov/ pubmed/28196961. Accessed 15 June 2020

29. Tanner MA, Goebel BM, Dojka MA, Pace NR. Specific ribosomal DNA sequences from diverse environmental settings correlate with experimental contaminants. Appl Environ Microbiol. 1998;64:3110-3 Available from: http://www.ncbi.nlm.nih.gov/pubmed/9687486.

30. Salter SJ, Cox MJ, Turek EM, Calus ST, Cookson WO, Moffatt MF, et al. Reagent and laboratory contamination can critically impact sequence-based microbiome analyses. BMC Biol. 2014;12:87 Available from: http://www.ncbi. nlm.nih.gov/pubmed/25387460.
31. Dickson RP. The lung microbiome and ARDS. It is time to broaden the model. Am J Respir Crit Care Med. 2018;197:549-51 Available from: http:// www.ncbi.nlm.nih.gov/pubmed/29091746.

32. Kitsios GD, Morowitz MJ, Dickson RP. Huffnagle GB, McVerry BJ, Morris A. Dysbiosis in the intensive care unit: microbiome science coming to the bedside. J Crit Care. 2017:38:84-91.

33. Navas-Molina JA, Peralta-Sánchez JM, González A, McMurdie PJ, VázquezBaeza $Y, X u Z$, et al. Advancing our understanding of the human microbiome using QIIME. Methods Enzymol. 2013;531:371-444 Available from: http://www.ncbi.nlm.nih.gov/pubmed/24060131.

34. Zhang Y, Ji P, Wang J, Zhao F. RiboFR-Seq: a novel approach to linking 165 rRNA amplicon profiles to metagenomes. Nucleic Acids Res. 2016;44:e99 Available from http://www.ncbi.nlm.nih.gov/pubmed/26984526.

35. Barfod KK, Roggenbuck M, Hansen LH, Schjørring S, Larsen ST, Sørensen SJ, et al. The murine lung microbiome in relation to the intestinal and vaginal bacterial communities. BMC Microbiol. 2013;13:303 Available from: http:// www.ncbi.nlm.nih.gov/pubmed/24373613.

36. Ren Y, Su H, She Y, Dai C, Xie D, Narrandes S, et al. Whole genome sequencing revealed microbiome in lung adenocarcinomas presented as ground-glass nodules. Transl Lung Cancer Res. 2019;8:235-46 Available from http://www.ncbi.nlm.nih.gov/pubmed/31367537.

37. Fox GE, Magrum $L$, Balch WE, Wolfe RS, Woese CR. Classification of methanogenic bacteria by 165 ribosomal RNA characterization. Proc Natl Acad Sci U S A. 1977;74:4537-41 Available from http://www.ncbi.nlm.nih. gov/pubmed/16592452.

38. Balch WE, Fox GE, Magrum LI, Woese CR, Wolfe RS. Methanogens: reevaluation of a unique biological group. Microbiol Rev. 1979;43:260-96 Available from http://www.ncbi.nlm.nih.gov/pubmed/390357.

39. Langelier C, Kalantar KL, Moazed F, Wilson MR, Crawford ED, Deiss T, et al. Integrating host response and unbiased microbe detection for lower respiratory tract infection diagnosis in critically ill adults. Proc Natl Acad Sci. 2018;115:E12353-62 Available from: http://www.pnas.org/lookup/doi/10.1 073/pnas.1809700115.

40. Rissin DM, Kan CW, Campbell TG, Howes SC, Fournier DR, Song L, et al. Single-molecule enzyme-linked immunosorbent assay detects serum proteins at subfemtomolar concentrations. Nat Biotechnol. 2010;28:595-9 Available from http://www.ncbi.nlm.nih.gov/pubmed/20495550.

41. Ma W, Kuang $H, X u L$, Ding L, Xu C, Wang L, et al. Attomolar DNA detection with chiral nanorod assemblies. Nat Commun. 2013;4:2689 Available from: http://www.ncbi.nlm.nih.gov/pubmed/24162144.

42. Lee J, Zhou M, Zhu H, Nidetz R, Kurabayashi K, Fan X. Fully automated portable comprehensive 2-dimensional gas chromatography device. Anal Chem. 2016;88:10266-74 Available from: http://www.ncbi.nlm.nih.gov/ pubmed/27709906.

43. van Oort PMP, Nijsen T, Weda $H$, Knobel H, Dark P, Felton T, et al. BreathDx - molecular analysis of exhaled breath as a diagnostic test for ventilatorassociated pneumonia: protocol for a European multicentre observational study. BMC Pulm Med. 2017;17:1 Available from: http://www.ncbi.nlm.nih. gov/pubmed/28049457.

44. Kyo M, Nishioka K, Nakaya T, Kida Y, Tanabe Y, Ohshimo S, et al. Unique patterns of lower respiratory tract microbiota are associated with inflammation and hospital mortality in acute respiratory distress syndrome. Respir Res. 2019;20:246 Available from: http://www.ncbi.nlm.nih.gov/ pubmed/31694652.

45. Dickson RP, Schultz MJ, van der Poll T, Schouten LR, Falkowski NR, Luth JE, et al. Lung microbiota predict clinical outcomes in critically ill patients. Am J Respir Crit Care Med. 2020; Available from: http://www.ncbi.nlm.nih.gov/ pubmed/31973575. Accessed 15 June 2020.

46. Wypych TP, Wickramasinghe LC, Marsland BJ. The influence of the microbiome on respiratory health. Nat Immunol. 2019;20:1279-90 Available from http://www.ncbi.nlm.nih.gov/pubmed/31501577.

47. Mandell LA, Niederman MS. Aspiration pneumonia. Longo DL, editor. N. Engl. J. Med. Massachussetts Medical Society; 2019. p. 651-63. Available from: http://www.nejm.org/doi/10.1056/NEJMra1714562. [cited 2020 Jun 4].

48. Zakharkina T, Martin-Loeches I, Matamoros S, Povoa P, Torres A, Kastelijn JB, et al. The dynamics of the pulmonary microbiome during mechanical ventilation in the intensive care unit and the association with occurrence of pneumonia. Thorax. 2017:72(9):803-10. https://doi.org/10.1136/thoraxinl2016-209158. Epub 2017 Jan 18.

49. Emonet S, Lazarevic V, Leemann Refondini C, Gaïa N, Leo S, Girard M, et al. Identification of respiratory microbiota markers in ventilator-associated 
pneumonia. Intensive Care Med. 2019;45(8):1082-92. https://doi.org/10.1007/ s00134-019-05660-8. Epub 2019 Jun 17.

50. Madan JC, Salari RC, Saxena D, Davidson L, OToole GA, Moore JH, et al. Gut microbial colonisation in premature neonates predicts neonatal sepsis. Arch Dis Child - Fetal Neonatal Ed. 2012;97:F456-62 Available from: http://fn.bmj. com/lookup/doi/10.1136/fetalneonatal-2011-301373.

51. Otani S, Chihade DB, Coopersmith CM. Critical illness and the role of the microbiome. Acute Med Surg. 2019;6:91-4 Available from: https:// onlinelibrary.wiley.com/doi/abs/10.1002/ams2.383.

52. Souza DG, Vieira AT, Soares AC, Pinho V, Nicoli JR, Vieira LQ, et al. The essential role of the intestinal microbiota in facilitating acute inflammatory responses. J Immunol. 2004;173:4137-46 Available from: http://www. jimmunol.org/lookup/doi/10.4049/jimmunol.173.6.4137.

53. Hranjec T, Rosenberger LH, Swenson B, Metzger R, Flohr TR, Politano AD, et al. Aggressive versus conservative initiation of antimicrobial treatment in critically ill surgical patients with suspected intensive-care-unit-acquired infection: a quasi-experimental, before and after observational cohort study. Lancet Infect Dis. 2012;12:774-80 Available from http://www.ncbi.nlm.nih. gov/pubmed/22951600.

54. Mukherjee S, Hanidziar D. More of the gut in the lung: how two microbiomes meet in ARDS. Yale J Biol Med. 2018;91:143-9 Available from: http://www.ncbi.nlm.nih.gov/pubmed/29955219.

55. Frisbee $A L$, Petri WA. Considering the immune system during fecal microbiota transplantation for Clostridioides difficile infection. Trends Mol Med. 2020;26(5):496-507. https://doi.org/10.1016/j.molmed.2020.01.009. Epub 2020 Feb 17.

56. Tavoukjian $\vee$. Faecal microbiota transplantation for the decolonization of antibiotic-resistant bacteria in the gut: a systematic review and metaanalysis. J Hosp Infect. 2019;102(2):174-88. https://doi.org/10.1016/j.jhin.2019. 03.010. Epub 2019 Mar 26.

57. Critically ill patients demonstrate large interpersonal variation in intestinal microbiota dysregulation: a pilot study. Intensive Care Med. 2016;43:59-68.

58. Alverdy JC, Krezalek MA. Collapse of the microbiome, emergence of the pathobiome, and the immunopathology of sepsis. Crit Care Med. 2017;45: 337-47 Available from: http://www.ncbi.n/m.nih.gov/pubmed/28098630.

59. Johnstone J, Heels-Ansdell D, Thabane L, Meade M, Marshall J, Lauzier F, et al. Evaluating probiotics for the prevention of ventilator-associated pneumonia: a randomised placebo-controlled multicentre trial protocol and statistical analysis plan for PROSPECT. BMJ Open. 2019;9:e025228 Available from http://www.ncbi.nlm.nih.gov/pubmed/31227528.

60. Freedberg DE, Zhou MJ, Cohen ME, Annavajhala MK, Khan S, Moscoso DI, et al. Pathogen colonization of the gastrointestinal microbiome at intensive care unit admission and risk for subsequent death or infection. Intensive Care Med. 2018;44:1203-11 Available from http://www.ncbi.nlm.nih.gov/ pubmed/29936583.

61. lapichino G, Callegari ML, Marzorati S, Cigada M, Corbella D, Ferrari S, et al. Impact of antibiotics on the gut microbiota of critically ill patients. J Med Microbiol. 2008;57:1007-14 Available from: http://www.ncbi.nlm.nih.gov/ pubmed/18628503.

62. Dickson RP, Singer BH, Newstead MW, Falkowski NR, Erb-Downward JR, Standiford TJ, et al. Enrichment of the lung microbiome with gut bacteria in sepsis and the acute respiratory distress syndrome. Nat Microbiol. 2016;1: 16113 Available from http://www.ncbi.nlm.nih.gov/pubmed/27670109.

63. Kristensen NB, Bryrup T, Allin KH, Nielsen T, Hansen TH, Pedersen O. Alterations in fecal microbiota composition by probiotic supplementation in healthy adults: a systematic review of randomized controlled trials. Genome Med. 2016;8:52 Available from http://www.ncbi.nlm.nih.gov/pubmed/2715 9972.

64. van Ruissen MCE, Bos LD, Dickson RP, Dondorp AM, Schultsz C, Schultz MJ. Manipulation of the microbiome in critical illness-probiotics as a preventive measure against ventilator-associated pneumonia. Intensive Care Med Exp. 2019;7:37 Available from http://www.ncbi.nlm.nih.gov/pubmed/31346841.

65. Weng H, Li J-G, Mao Z, Feng Y, Wang C-Y, Ren X-Q, et al. Probiotics for preventing ventilator-associated pneumonia in mechanically ventilated patients: a meta-analysis with trial sequential analysis. Front Pharmacol. 2017;8:717 Available from http://www.ncbi.nlm.nih.gov/pubmed/29062279.

66. Morrow LE, Wischmeyer P. Blurred lines: dysbiosis and probiotics in the ICU. Chest. 2017;151:492-9 Available from: http://www.ncbi.nlm.nih.gov/ pubmed/27771302.

67. Buelow E, Gonzalez TB, Versluis D, Oostdijk EAN, Ogilvie LA, van Mourik MSM, et al. Effects of selective digestive decontamination (SDD) on the gut resistome. J Antimicrob Chemother. 2014;69:2215-23 Available fromhttp:// www.ncbi.nlm.nih.gov/pubmed/24710024.

68. Wittekamp BH, Plantinga NL, Cooper BS, Lopez-Contreras J, Coll P, Mancebo $J$, et al. Decontamination strategies and bloodstream infections with antibiotic-resistant microorganisms in ventilated patients: a randomized clinical trial. JAMA. 2018;320:2087-98 Available fromhttp://www.ncbi.nlm.nih. gov/pubmed/30347072

69. de Smet AMGA, Kluytmans JAJW, Cooper BS, Mascini EM, Benus RFJ, van der Werf TS, et al. Decontamination of the digestive tract and oropharynx in ICU patients. N Engl J Med. 2009;360:20-31.

70. Goldstein EJC, Tyrrell KL, Citron DM. Lactobacillus species: taxonomic complexity and controversial susceptibilities. Clin Infect Dis. 2015;60(Suppl 2):S98-107 Available fromhttp://www.ncbi.n/m.nih.gov/pubmed/25922408.

71. Furusawa Y, Obata Y, Fukuda S, Endo TA, Nakato G, Takahashi D, et al. Commensal microbe-derived butyrate induces the differentiation of colonic regulatory T cells. Nature. 2013;504:446-50 Available from http://www.ncbi. nlm.nih.gov/pubmed/24226770.

72. Ng KM, Ferreyra JA, Higginbottom SK, Lynch JB, Kashyap PC, Gopinath S, et al. Microbiota-liberated host sugars facilitate post-antibiotic expansion of enteric pathogens. Nature. 2013;502:96-9 Available from http://www.ncbi. nlm.nih.gov/pubmed/23995682.

73. Wu H, Esteve E, Tremaroli V, Khan MT, Caesar R, Mannerås-Holm L, et al. Metformin alters the gut microbiome of individuals with treatment-naive type 2 diabetes, contributing to the therapeutic effects of the drug. Nat Med. 2017;23:850-8 Available from http://www.ncbi.nlm.nih.gov/pubmed/2 8530702.

74. Segal LN, Clemente JC, Wu BG, Wikoff WR, Gao Z, Li Y, et al. Randomised, double-blind, placebo-controlled trial with azithromycin selects for antiinflammatory microbial metabolites in the emphysematous lung. Thorax. 2017;72:13 LP-22 Available from: http://thorax.bmj.com/content/72/1/13. abstract.

75. Lax S, Gilbert JA. Hospital-associated microbiota and implications for nosocomial infections. Trends Mol Med. 2015;21:427-32 Available from http://www.ncbi.nlm.nih.gov/pubmed/25907678.

76. Kaul R, Burt JA, Cork L, Dedier H, Garcia M, Kennedy C, et al. Investigation of a multiyear multiple critical care unit outbreak due to relatively drugsensitive Acinetobacter baumannii: risk factors and attributable mortality. J Infect Dis. 1996;174:1279-87 Available from: http://www.ncbi.nlm.nih.gov/ pubmed/8940219.

\section{Publisher's Note}

Springer Nature remains neutral with regard to jurisdictional claims in published maps and institutional affiliations. 